Ocular Oncology

and Pathology
Ocul Oncol Pathol 2017;3:95-100

DOI: $10.1159 / 000450858$
Received: May 19, 2016

Accepted after revision: Sept, ember 13, 2016

Published online: November 8, 2016

\title{
Extramedullary Relapse of Acute Myelogenous Leukemia Presenting as a Large Serous Retinal Detachment
}

\author{
Wesley Green ${ }^{a}$ P. Kumar Rao ${ }^{a}$ George J. Harocopos ${ }^{a}$ b \\ Departments of a Ophthalmology and Visual Sciences and ${ }^{\mathrm{b}}$ Pathology and Immunology, Washington University \\ School of Medicine, St. Louis, MO, USA
}

\section{Keywords}

Leukemia · Retinal detachment · Granulocytic sarcoma ·

Chloroma $\cdot$ Leukemic choroidal infiltration

\begin{abstract}
Background/Aims: To describe the rare presentation of a large, unilateral, serous retinal detachment as an extramedullary manifestation of acute myelogenous leukemia (AML) recurrence without bone marrow or central nervous system involvement after more than 1 year of follow-up. Methods: Case report. Results: A teenage patient with $\mathrm{AML}$, previously treated with multiple courses of systemic chemotherapy, radiation, and bone marrow transplant, presented with acute vision loss. Ophthalmic workup revealed a large, unilateral, bullous, serous retinal detachment. Ultimately, he underwent subretinal fluid biopsy, which was found to be positive for leukemic blast cells. Cytologic markers matched his initial bone marrow biopsy, and therefore were diagnostic of extramedullary AML relapse. Conclusions: Leukemia can cause various ophthalmic manifestations. Autopsy studies suggest that choroidal infiltration is relatively common, but clinical progression to serous retinal detachment is quite uncommon. Furthermore, serous retinal detachment is generally shallow, posterior, and much more often reported in
\end{abstract}

\section{KARGER}

(c) 2016 S. Karger AG, Basel

E-Mail karger@karger.com

www.karger.com/oop acute lymphocytic leukemia. The ophthalmologist plays a critical role in identifying leukemic ocular involvement. This case demonstrates the potential for ocular biopsy to secure the diagnosis of extramedullary relapse in order to initiate prompt treatment and systemic workup.

(c) 2016 S. Karger AG, Base

\section{Introduction}

Leukemia is a broad term describing systemic malignancies that develop in the blood-forming tissues (i.e., bone marrow). The major categories of leukemia include acute myelogenous leukemia (AML), acute lymphoblastic leukemia (ALL), chronic myelogenous leukemia, and chronic lymphocytic leukemia. Of all new cancer diagnoses, leukemia represents approximately $3.3 \%$, or just over 54,000 new cases, as of the year 2015. Over the last 40 years, significant advances in management and treatment of leukemia have increased the 5-year survival from just over $30 \%$ in 1975 to roughly $60 \%$ today, while the number of new cases has remained relatively stable over that same time period $[1,2]$.

Ophthalmic manifestations of leukemia are protean and numerous. Ocular involvement can occur from di- 
rect leukemic invasion, indirect systemic effects from the malignancy (i.e., hyperviscosity, anemia, or thrombocytopenia), or from local or systemic treatment effects (i.e., chemotherapy, radiation, or bone marrow transplant [BMT]) [3]. Estimates of the incidence of ocular manifestations in leukemia range from approximately 25 to $65 \%$ of cases [4-6]. Clinically, direct leukemic tissue invasion is not commonly apparent, while secondary or indirect effects of the malignancy or treatment are more readily identified on clinical examination. Overall, manifestations are more common in acute versus chronic, and in myelogenous versus lymphocytic leukemias [7]. We are presenting a case of acute myelogenous leukemia with a rare and somewhat unclear initial ocular presentation, which resulted in the ophthalmology team playing a critical role in identifying leukemic relapse. Furthermore, we retrospectively evaluate and discuss whether a seemingly unrelated periocular manifestation may have been an initial sign of what was to follow.

\section{Case Report}

Our patient was a 14-year-old African American male at the time of initial diagnosis of AML with minimal differentiation (French-American-British classification subtype M0) on bone marrow biopsy at the end of 2011. Cytochemistries on the bone marrow biopsy were negative for myeloperoxidase and naphthyl butyrate esterase. On flow cytometry, the blast cells expressed CD13, CD33, CD56, and CD117, but not CD15. B-cell markers (CD10, CD19, CD20, cytoplasmic CD79a, surface kappa and lambda light chains) and T-cell markers (CD1a, $\mathrm{CD} 2$, surface $\mathrm{CD} 3$, $\mathrm{CD} 4, \mathrm{CD} 5$, and CD8) were negative, as were TdT and cytoplasmic $\mathrm{CD} 3$, thereby excluding B-lymphoblastic, T-lymphoblastic, and NK leukemias. The diagnosis of AML was further substantiated with fluorescence in situ hybridization, which revealed a clonal interstitial deletion of chromosome $5 \mathrm{q}$ (characteristic of AML), among other anomalies. Cerebrospinal fluid analysis was not consistent with central nervous system involvement, and the patient underwent induction chemotherapy. In early 2012, he had an initial relapse and underwent salvage chemotherapy. Approximately 1 year later, in early 2013, he had another relapse and underwent additional salvage chemotherapy. In July 2013, he underwent haploidentical BMT after 200-cGy whole-body irradiation. He had an unremarkable pre-BMT ophthalmological evaluation.

The patient was well for 14 months following BMT, until he developed severe holocephalic headaches in September 2014, for which he was admitted to the hospital for further workup. Head imaging with CT and MRI showed "chronic sinusitis." Lumbar puncture revealed normal opening pressure and cytology. The headaches were associated with light and sound sensitivity and were ultimately treated as migraines with short-term valproic acid. Approximately 1 month later, in October 2014, the patient was readmitted due to 3 days of increasing right periocular erythema and edema associated with vague ocular discomfort and minimal clear discharge. Ophthalmic exam showed a minimal decrease in

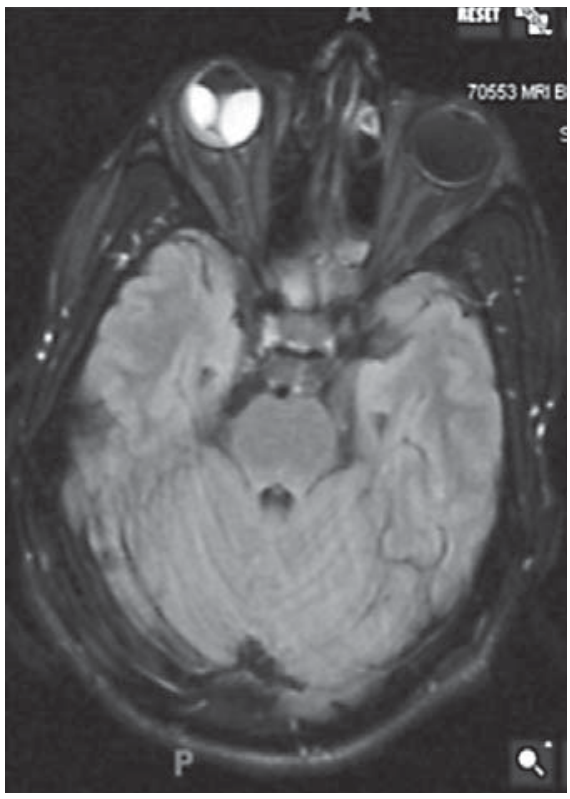

Fig. 1. Axial T2/FLAIR MRI image of the brain and orbits demonstrating T2 hyperintense bullous elevations of the retina versus choroid, with thickening of the choroid and/or sclera.

acuity to 20/25 on the right, equal pupils without relative afferent defect, moderate elevation of right-eye intraocular pressure (IOP) to 33 , trace limitation of supraduction on the right, equal and intact sensation to light touch in the trigeminal distributions, moderate periorbital skin erythema and edema on the right, along with moderate conjunctival injection most prominent temporally and trace subconjunctival hemorrhage nasally, and an otherwise unremarkable anterior segment and dilated examination. Clinically, this was felt to most likely represent preseptal cellulitis, although early orbital cellulitis was still not ruled out. Given his history of malignancy and potential immunocompromise, he was managed as an inpatient. He was placed on intravenous broad-spectrum antibiotics, as well as dorzolamide/timolol eyedrops twice daily to the right eye. Additionally, he was incorrectly taking $5 \mathrm{mg}$ oral prednisone daily as an outpatient, so this was concomitantly increased to his prescribed dose of $35 \mathrm{mg}$ daily. Orbital CT revealed preseptal inflammation, globe wall thickening, and worsening sinus disease compared to prior imaging, but no orbital involvement, cavernous sinus involvement, or significant sinus disease adjacent to the affected right side. He was followed closely in-house for 5 days with improvement in visual acuity, motility, IOP, periocular swelling, and conjunctival injection. He was discharged on oral antibiotics to complete a total course of 14 days, continued on his previously prescribed oral prednisone, and scheduled for outpatient follow-up.

He continued to do well until January of 2015, when he developed 3-4 days of increasing headache, right-eye redness and discomfort, and significantly decreased vision. MRI (Fig. 1) demonstrated T2 hyperintense bullous elevations of the retina versus choroid (at the time interpreted as choroidal effusion), with thickening of the choroid and/or sclera. Additionally, worsening sinus disease
Green/Rao/Harocopos 
2

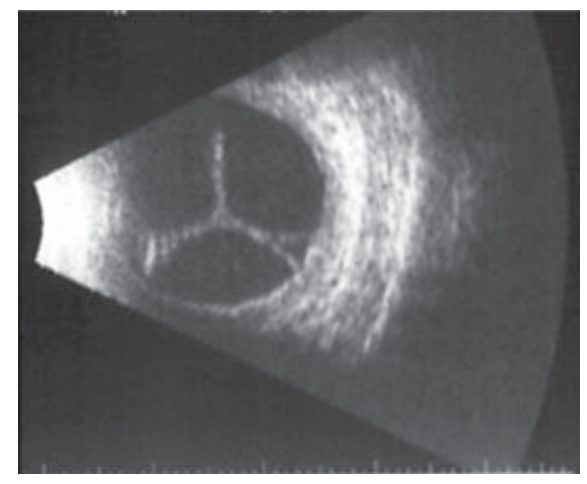

Fig. 2. B-scan ultrasound of the right eye demonstrating fluid in the subretinal versus suprachoroidal space.

Fig. 3. Cytology of the subretinal fluid biopsy (original magnification, $\times 1,000$ ). a H\&E stain exhibiting several cells with relatively larger and in some cells irregular nuclei (arrows) consistent with leukemic blast cells. The cells with smaller nuclei are consistent with a mixture of chronic inflammatory cells (macrophages and mature lymphocytes, arrowheads). b CD163 immunostain highlighting macrophages. Note the negatively stained cells (arrows) consistent with leukemic blast cells. c CD56 immunostain confirming the presence of leukemic blast cells (arrows), supporting the diagnosis of ocular involvement by relapsing AML. Fig. 4. Composite of sequential Optos wide-field fundus photographs of the right eye from February (a), May (b), and October (c) 2015 showing progressive improvement of the bullous serous retinal detachment.
3

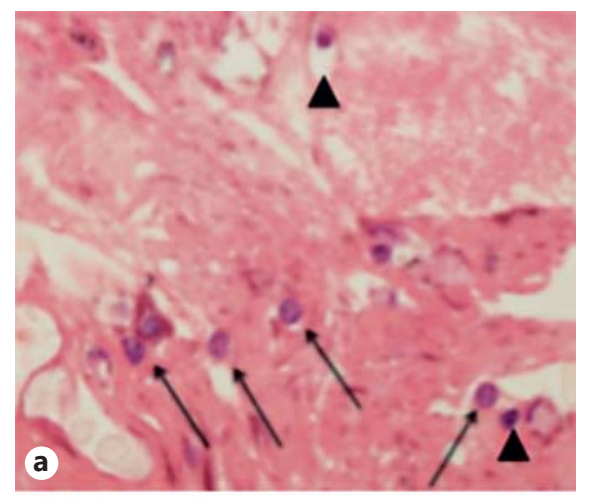

4
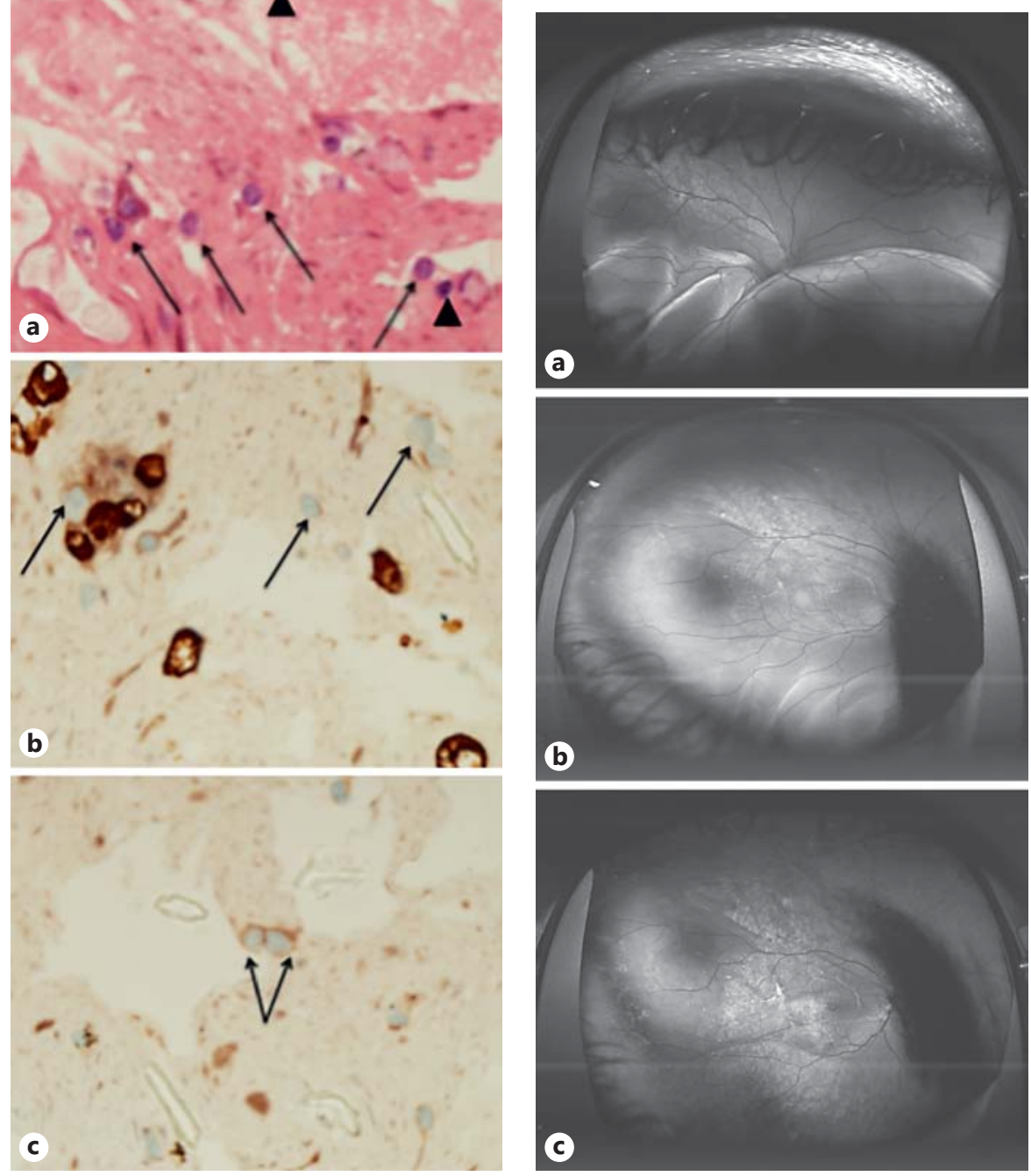

(b)
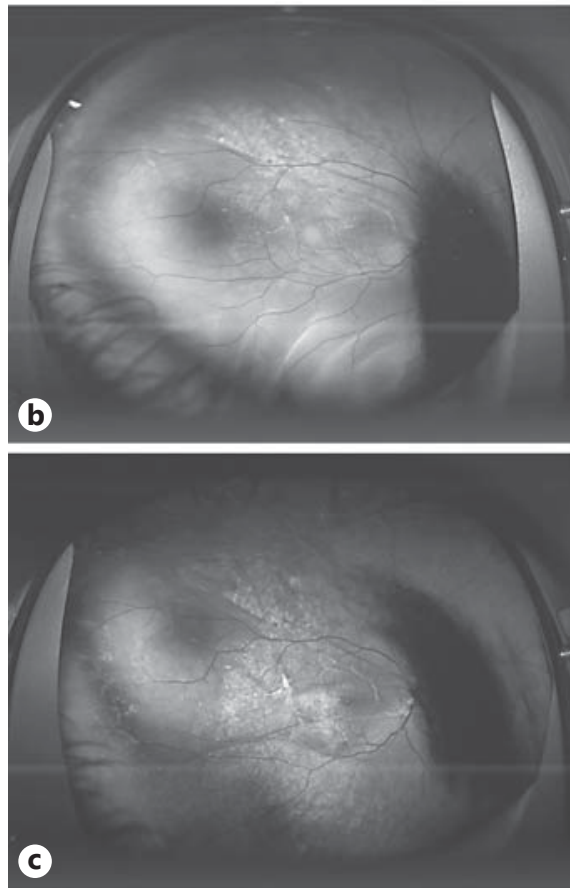

was observed in the sphenoid sinuses and left maxillary sinus opposite the affected eye. Ophthalmic examination showed light perception vision in the right eye with normal IOP, motility, and pupillary examination. External examination revealed no periocular swelling. Anterior segment showed moderate conjunctival injection without anterior chamber inflammation. Posterior visualization was limited, but B-scan ultrasound (Fig. 2) was consistent with MRI findings, at the time interpreted as "kissing choroidals" related to presumed posterior scleritis. Initial management with high-dose oral prednisone ( $60 \mathrm{mg}$ daily) was initiated. The patient had difficulty keeping up with the appropriate dosing, and no appreciable changes were noted on examination over 2 weeks of treatment. At that point, the possibility of ocular involvement by AML was entertained, and it was decided to proceed to the operating room to obtain a cytologic diagnosis.

In early February 2015, trans-scleral fine-needle aspiration biopsy was performed, with no complications, from what was initially presumed to be the suprachoroidal space. The fluid aspirate was noted grossly to appear yellow-colored. Histologic staining was critical for the clinical-pathological correlation and diagnosis (Fig. 3). Cytologic smears were prepared, in addition to a cell block (paraffin-embedded) for hematoxylin and eosin (H\&E) stain and immunohistochemistry. The biopsy fluid primarily contained macrophages scattered within proteinaceous fluid exudate, but both Pap and H\&E stain exhibited some cells with relatively large and irregular nuclei with coarse chromatin, consistent with blast cells. Negative Gram and Gomori methenamine silver stains, along with the dearth of neutrophils, ruled out infection. (A portion of the ocular fluid was also submitted for bacterial and fungal cultures, which were also negative.) CD68 and CD163 immunostains confirmed the abundance of macrophages, while the suspected blast cells were positive for CD56 and CD117 (c-kit) but negative for myeloperoxidase. (The blast cells were also positive for the cell proliferation marker Ki-67.) Similarly, flow cytometry performed on the initial bone marrow biopsy in late 2011 had demonstrated expression of the markers CD56 and CD117 in the leukemic cells, 
while cytochemistry for myeloperoxidase was negative. These findings therefore secured the diagnosis of extramedullary ocular recurrence of AML. Subsequently, the patient underwent a systemic evaluation, which included lumbar puncture, bone marrow biopsy, and CT imaging of the chest, abdomen, pelvis, and neck, all of which were unremarkable. He underwent intrathecal chemotherapy and 2,400-cGy fractionated radiotherapy to the right orbit in March 2015.

Over the ensuing 9 months, he continued to have a slow and consistent improvement in the posterior segment ocular abnormalities (Fig. 4) with visual acuity stabilizing around 20/400. As the posterior bullous elevations resolved, with improved visualization of the posterior segment, it became evident that these, from the outset, represented near-total exudative retinal detachment rather than "kissing" choroidal effusion (e.g., note retinal folds in Fig. 4a). Unfortunately, his systemic course remained much more tenuous. In July 2015, he was hospitalized with Streptococcus pneumoniae septic shock. Then in August, he had a return of migrainelike headaches and was later hospitalized again with severe pneumonia. Several imaging studies over this period showed more significant progression of sinus disease, which was ultimately biopsied via functional endoscopic sinus surgery in September 2015. Multiple specimens from the left nasal cavity and left posterior ethmoid sinus were positive for extramedullary recurrence of AML (i.e., granulocytic or myeloid sarcoma; chloroma), with several flow cytometric cell surface markers (CD33, CD117) matching those of the initial diagnostic bone marrow biopsy and also correlating with the immunohistochemical results of the ocular fluid biopsy (CD117-positive). After repeat systemic evaluation in October 2015, again negative for any identifiable systemic involvement, he received an additional 2,400-cGy fractionated radiotherapy to bilateral maxillary sinuses, bilateral ethmoid sinuses, bilateral sphenoid sinuses, left frontal sinus, and nasal cavity.

Approximately 2 months later, in December 2015, he was noted to have bilateral scrotal discomfort with masses noted on clinical examination and ultrasound. Prior to further outpatient management, he developed influenza A pneumonia with septic shock and was admitted to the intensive care unit. After systemic stabilization, he underwent brain and orbit MRI and paratesticular mass biopsy. He was found to have extramedullary AML relapse on the paratesticular biopsy specimen, which was again positive on flow cytometry for CD33 and CD117. MRI showed mild postradiation changes of the orbits and resolution of prior ocular abnormalities. As of the time of this writing, he has undergone additional repeat lumbar puncture and bone marrow biopsy, which remain negative for leukemic involvement. He has decided not to pursue any further local or systemic treatment.

\section{Discussion}

Returning to the presentation of acute vision loss with total exudative retinal detachment versus choroidal effusion, the leading diagnoses were posterior scleritis versus leukemic relapse. After biopsy and cytologic evaluation, it was clear that the diagnosis was extramedullary recurrence of AML. Both the MRI and B-scan findings initial- ly were interpreted as classic "kissing choroidals," but clinically there was no suprachoroidal hemorrhage, hypotony, or large IOP change to serve as a potential etiology for choroidal effusion. Assessing the presence of shifting subretinal fluid by performing B-scan in both upright and supine positions may have helped distinguish exudative retinal detachment from choroidal effusion at that time, but as per our review of the patient's office records, there is no documentation that this was attempted. In addition, optical coherence tomography would generally be useful in pinpointing the location of the fluid space, but the limited posterior visualization at the time may have precluded this. After confirmation of AML on cytology, B-scan and MRI images were retrospectively reviewed. When combining all aspects of the examination, imaging, and histopathology, we feel this presentation is consistent with choroidal thickening from direct leukemic cell invasion with secondary serous retinal detachment and leakage of leukemic cells into the subretinal space. Therefore, the imaging findings initially thought to represent a choroidal effusion actually showed a neartotal serous retinal detachment, which became especially evident during the resolution phase.

Despite retinopathy being the most commonly reported clinical ophthalmic manifestation [3-5], and despite rare reports of direct and necrotic retinal invasion [8], autopsy studies suggest that subclinical choroidal infiltration [9-11] is the most common form of ophthalmic involvement by leukemia. Serous retinal detachment as a leukemic manifestation is uncommon overall [12], and generally presents during active systemic disease, more commonly in ALL than in AML, and presents as a shallow detachment at the posterior pole, which was not the case in our patient. Less common still is serous retinal detachment as the presenting sign of recurrence. Of the 7 cases of ALL recurrence presenting with serous retinal detachment that we were able to identify in the literature, 4 [12-15] reported bone marrow and/or central nervous system involvement at the time of presentation or within 6 months; 1 [16] reported subsequent systemic involvement shortly after presentation, but did not report a specific time frame; 1 [17] reported no systemic involvement immediately upon presentation, but gave no follow-up; and 1 [18] specifically reported the lack of systemic involvement through 18 months of follow-up.

Literature-reported cases of AML recurrence presenting with serous retinal detachment are exceedingly rare $[19,20]$. Wu et al. [19] reported a patient with bilateral serous retinal detachment and concurrent bone marrow AML recurrence. Uozumi et al. [20] reported an AML
Green/Rao/Harocopos 
recurrence with a shallow, posterior, serous retinal detachment in a patient who remained free of systemic disease until bone marrow recurrence 12 months after initial presentation. To the best of our knowledge, after extensive literature review, our patient appears to be the first reported case of AML recurrence presenting with a large, unilateral, serous retinal detachment with follow-up greater than 1 year without evidence of bone marrow or central nervous system involvement.

A review of our patient's initial ophthalmic presentation with presumed preseptal cellulitis potentially represents another uncommon presentation of his neoplastic disease. On the one hand, the clinical appearance was fairly consistent with preseptal cellulitis, and it appeared to respond after introduction of antibiotic treatment. On the other hand, there were several factors that complicated the presentation. First, when he presented, he was taking only $5 \mathrm{mg}$ of oral prednisone daily, instead of the $35 \mathrm{mg}$ prescribed as an outpatient. Upon admission, this was restarted at full dose concurrently with the initiation of antibiotics. Therefore, it is not entirely clear which of these medications served as the primary treatment. Secondly, there was imaging evidence of globe wall thickening in addition to the expected preseptal inflammation. Furthermore, there was no evidence of sinusitis adjacent to the involved eye and no clinical findings or reported history of insect bite, animal scratch, or other local skin trauma to serve as a site of entry for microbes. Given the leukemic choroidal invasion that subsequently presented as a serous retinal detachment on the same side shortly thereafter, we believe these findings may have been a pseudo-cellulitis representing initial leukemic invasion instead of a reactive inflammatory change associated with preseptal cellulitis. (The subsequent diagnosis of granulocytic sarcoma on endoscopic sinus biopsy lends further credence to this hypothesis.) Cutaneous granulocytic sarcoma, termed leukemia cutis, is infiltration of neoplastic granulocytes into the epidermis, dermis, or subcutaneous tissues. Alternatively, Sweet's syndrome (i.e., acute febrile neutrophilic dermatosis), sometimes associated with acute leukemia, is infiltration of mature neutrophils, but is generally associated with fever, headache, malaise, and other systemic symptoms [21]. Granulocytic sarcoma presenting as periocular swelling/inflammation mimicking preseptal cellulitis has been reported in the literature [21-23], yet this possibility was not considered at the time, and definitive diagnosis requires cutaneous biopsy, which was not obtained in our case. Finally, regardless of whether he truly had either leukemia cutis or Sweet's syndrome, another unusual aspect of this case is the multifo- cal nature (eye, paranasal sinuses, testicles) of the subsequent granulocytic sarcomas separated by space and time, which were not associated with active bone marrow disease at the time of diagnosis.

\section{Conclusions}

Leukemia can result in various ocular and periocular manifestations, including serous retinal detachment associated with leukemic choroidal infiltration, paranasal sinus infiltration, and leukemia cutis. Autopsy studies suggest that choroidal infiltration is relatively common, but clinical progression to serous retinal detachment is quite uncommon. Furthermore, serous retinal detachment is generally shallow, posterior, and much more often reported in acute lymphocytic leukemia. The ophthalmologist plays a critical role in identifying leukemic ocular involvement. Finally, this case demonstrates the potential for ocular biopsy to secure the diagnosis of extramedullary relapse in order to initiate prompt treatment and systemic workup. As of the writing of this paper, this patient has remained free from identifiable bone marrow involvement approximately 14 months after initial ocular relapse.

\section{Statement of Ethics}

This study was conducted in compliance with the Health Insurance Portability and Accountability Act.

\section{Disclosure Statement}

The authors declare that there are no financial conflicts of interest.
References
1 SEER Cancer Statistics Factsheets: Leukemia National Cancer Institute. Bethesda, MD. http://seer.cancer.gov/statfacts/html/leuks. html.
2 Howlader N, Noone AM, Krapcho M, Garshell J, Miller D, Altekruse SF, Kosary CL, Yu M, Ruhl J, Tatalovich Z, Mariotto A, Lew- is DR, Chen HS, Feuer EJ, Cronin KA (eds): SEER Cancer Statistics Review, 1975-2012, National Cancer Institute. Bethesda, MD. http://seer.cancer.gov/csr/1975_2012/ (based on November 2014 SEER data submission, posted to the SEER website, April 2015). 
3 Sharma T, Grewal J, Gupta S, Murray PI: Ophthalmic manifestations of acute leukemias: the ophthalmologist's role. Eye 2004;18: 663-672.

4 Russo V, Scott IU, Querques G, Stella A, Barone A, Delle Noci N: Orbital and ocular manifestations of acute childhood leukemia: clinical and statistical analysis of 180 patients. Eur J Ophthalmol 2008;18:619-623.

5 Alemayehu W, Shamebo M, Bedri A, Mengistu Z: Ocular manifestations of leukaemia in Ethiopians. Ethiop Med J 1996;34:217-224.

6 Karesh JW, Goldman EJ, Reck K, Kelman SE, Lee EJ, Schiffer CA: A prospective ophthalmic evaluation of patients with acute myeloid leukemia: correlation of ocular and hematologic findings. J Clin Oncol 1989;7:1528-1532.

7 Koshy J, John MJ, Thomas S, Kaur G, Batra N, Xavier WJ: Ophthalmic manifestations of acute and chronic leukemias presenting to a tertiary care center in India. Indian J Ophthalmol 2015;63:659-664.

8 Primack JD, Smith ME, Tychsen L: Retinal detachment in a child as the first sign of leukemic relapse: histopathology, MRI findings, treatment, and tumor-free follow up. J Pediatr Ophthalmol Strabismus 1995;32:253-256.

9 Allen RA, Straatsma BR: Ocular involvement in leukemia and allied disorders. Arch Ophthalmol 1961;66:490-508.

10 Robb RM, Ervin LD, Sallan SE: An autopsy study of eye involvement in acute leukemia of childhood. Med Pediatr Oncol 1979;6: 171-177.
11 Leonardy NJ, Rupani M, Dent G, Klintworth GK: Analysis of 135 autopsy eyes for ocular involvement in leukemia. Am J Ophthalmol 1990;109:436-444.

12 Miyamoto K, Kashii S, Honda Y: Serous retinal detachment caused by leukemic choroidal infiltration during complete remission. $\mathrm{Br} \mathrm{J}$ Ophthalmol 2000;84:1318.

13 Schmiegelow K, Scherfig E, Prause JU, Jensen OA: Isolated leukemic choroidal relapse in a child with acute lymphoblastic leukemia one year off therapy, diagnosed through transvitreal retino-choroidal biopsy. Acta Ophthalmol (Copenh) 1988;66:33-37.

14 Subramanyam A, Phatak S, Chandalia S, Kane SV, Banavali SD: Ocular biopsy through external needle drainage for isolated bilateral ocular relapse of childhood acute lymphoblastic leukemia. Retin Cases Brief Rep 2011; 5:333-335.

15 Golan S, Goldstein M: Acute lymphocytic leukemia relapsing as bilateral serous retinal detachment: a case report. Eye (Lond) 2011;25: 1375-1378.

16 Yoshida K, Hasegawa D, Takusagawa A, Kato I, Ogawa C, Echizen N, Ohkoshi K, Yamaguchi T, Hosoya R, Manabe A: Bullous exudative retinal detachment due to infiltration of leukemic cells in a child with acute lymphoblastic leukemia. Int J Hematol 2010;92: 535-537.
17 Johnson JS, Lopez JS, Kavanaugh AS, Liang C, Mata DA: A 25-year-old man with exudative retinal detachments and infiltrates without hematological or neurological findings found to have relapsed precursor T-cell acute lymphoblastic leukemia. Case Rep Ophthalmol 2015;6:321-327.

18 Azik FM, Akinci A, Sayli TR, Culha VK, Teberik K, Teke MY, Gurbuz F: Unilateral exudative retinal detachment as the sole presentation of relapsing acute lymphoblastic leukemia. Turk J Haematol 2012;29:181-184.

$19 \mathrm{Wu}$ L, Calderon M, Hernandez G, Marbis J, Ramirez V: Bilateral exudative retinal detachment as the first sign of relapsing acute myelogenous leukaemia. Clin Experiment Ophthalmol 2006;34:623-625.

20 Uozumi K, Takatsuka Y, Ohno N, Hanada S, Tabata Y, Arimura H, Nakao K, Arima T: Isolated choroidal leukemic infiltration during complete remission. Am J Hematol 1997;55: 164-165.

21 Morgan KW, Callen JP: Sweet's syndrome in acute myelogenous leukemia as periorbital cellulitis with an infiltrate of leukemic cells. J Am Acad Dermatol 2001;45:590-595.

22 Bagheri A, Abrishami A, Karimi S: Acute myelogenous leukemia mimicking fulminant periorbital cellulitis. J Ophthalmic Vis Res 2013;8:380-382.

23 Takaue Y, Culbert SJ, van Eys J, Dalton WT Jr, Cork A, Trujillo JM: Spontaneous cure of end-stage acute nonlymphocytic leukemia complicated with chloroma (granulocytic sarcoma). Cancer 1986;58:1101-1105. 